\title{
3 Research Suare \\ Trehalose and glucose levels regulate feeding behaviour in two color morphs of Acyrthosiphon pisum Harris
}

\section{Guang Wang}

Gansu Agricultural University

Jing-Jiang Zhou

Gansu Agricultural University

Yan Li

Gansu Agricultural University

Yuping Gou

Gansu Agricultural University

Peter Quandahor

Gansu Agricultural University

Changzhong Liu ( $\square$ liuchzh@gsau.edu.cn )

Gansu Agricultural University

\section{Research Article}

Keywords: feeding behaviour, Acyrthosiphon pisum, EPG, trehalose, glucose, physiological sugar

Posted Date: February 16th, 2021

DOl: https://doi.org/10.21203/rs.3.rs-198698/v1

License: (9) (i) This work is licensed under a Creative Commons Attribution 4.0 International License.

Read Full License

Version of Record: A version of this preprint was published at Scientific Reports on August 5th, 2021. See the published version at https://doi.org/10.1038/s41598-021-95390-z. 


\section{Abstract}

Trehalose serves multifarious roles in growth and development in insects. We have previously shown that trehalose regulates Acyrthosiphon pisum chitin metabolism. Accordingly, we hypothesize here that trehalose-regulated $A$. pisum responses in chitin metabolism might also include trehalose-regulated feeding behaviour to involve in chitin metabolism. When RNA interference (RNAi) of trehalose-6phosphate synthase gene increased the percentage of E2 (i.e. phloem ingestion) waveform and decreased the percentage of $\mathrm{F}$ (i.e. stylet work) and $\mathrm{G}$ (i.e. xylem ingestion) waveforms compared with the control A. pisum. RNAi of trehalase gene did not affect the percentage of each waveform compared with the control A. pisum. The high trehalose and glucose diets increased the percentage of E2 waveform of red $A$. pisum. The multiple nonlinear regression shown that the both low trehalose and glucose levels increased the percentage of $\mathrm{np}$ (i.e. non-probing), E1 (i.e. phloem salivation), and E2 waveforms. The high or low trehalose:glucose $(T: G)$ ratio decreased the percentage of $n p, E 1$, and E2. Interestingly, the percentage of $\mathrm{C}$ (i.e. probing), $\mathrm{F}$ and, $\mathrm{G}$ waveforms were increased at low, low, and high $\mathrm{T}: \mathrm{G}$ ratio, respectively. The results provided strong evidence that the trehalose and glucose levels regulate $A$. pisum feeding behavior.

\section{Background}

Understanding insect feeding behaviours is important for insect pest management. Previous studies have shown that insect feeding behaviour is strongly influenced by biotic and abiotic factors ${ }^{1-5}$, as well as by the change in its physiology status, host plant nutrition and species ${ }^{6}$, and resistance to pesticide ${ }^{3,7}$. Aphids use their stylets to obtain nutrients from sieve tubes of plant tissue, and ingest passively on the phloem, driven by the pressure in the sieve tubes, and actively on the xylem, intercellular apoplastic and epidermal ${ }^{8}$. The stylet penetrates into the plant tissue and forms a stable food channel with secreting saliva to ingest plant sap ${ }^{9}$. The electrical penetration graph (EPG) has been used to monitor stylet activity, saliva excretion and food ingestion during aphid feeding recorded as different EPG waveforms associated with specific stylet tip positions and activities ${ }^{3,7,10}$. The EPG waveform np, C and E1, represent, respectively, non-probing, intercellular apoplastic stylet pathway and salivation into phloem sieve elements at the beginning of the phloem phase ${ }^{4,7}$. While the EPG waveform E2, G and F are correlated with passive phloem sap uptake from sieve element, active intake of xylem sap and derailed stylet mechanics, respectively ${ }^{4,7}$. Interestingly, it was reported that aphids aposymbiotics (endosymbiotic bacteria Buchnera aphidicola disruption), pesticides and pathogen Pandora neoaphidis affected feeding behaviours of piercing-sucking insects ${ }^{3-5,7}$. However, the studies of the effects of body sugar level on aphid feeding behaviours and EPG waveforms are very limited.

Sugar such as trehalose, a non-reducing disaccharide in insect hemolymph, is formed by two glucose molecules linked by an $a-a$ bond and widely present in bacteria, fungi, insects, and plants ${ }^{11-13}$. It is mainly present in hemolymph of insects and typically occurs at a high concentration; whereas glucose may occur together with trehalose, but at a significantly lower concentration ${ }^{14}$. Trehalose plays important roles 
in the growth, development ${ }^{15,16}$, flight, feeding ${ }^{17,18}$, overwinter and diapause ${ }^{19}$ of insects. Simpson and Raubenheimer $(1993)^{20}$ suggested that hemolymph trehalose level reflects the nutritional status of the insect and may serve a role in regulating food choice and nutrient consumption. Dietary nutrient levels on gluconeogenesis in Manduca sexta was positively correlated to hemolymph trehalose levels ${ }^{21}$, and the ratio of carbon to nitrogen from carbohydrate absorption affected the growth and development of Acyrthosiphon pisum ${ }^{2,22}$. A growing number of studies have found that elevation of trehalose rich has great agronomic potential to improve the stress tolerance of plants ${ }^{23,24}$. Trehalose serves multifarious roles in insects such as facilitating carbohydrate absorption, being a source of energy, and a component of a feedback mechanism regulating feeding behaviour and nutrient intake ${ }^{13,25}$. However, the feedback mechanism of rich trehalose on the feeding behaviours of piercing-sucking insects have not been clarified yet.

It is well known that trehalose-6-phosphate synthase (TPS) and trehalase (TRE) can directly or indirectly affect trehalose content and feeding behaviour ${ }^{16,26-28}$. Knockdown of TRE genes increased trehalose content and reduced food intake of $S$. exigua ${ }^{26}$, while knockdown of TPS reduced trehalose content but did not affect feeding behaviour of Nilaparvata lugens ${ }^{16} \square$ Bactrocera minax ${ }^{28}$ and Leptinotarsa decemlineata ${ }^{27}$. We have recently shown that RNAi of ApTPS and ApTRE regulate the chitin metabolism of $A$. pisum ${ }^{29}$. However, the study on the TPS and TRE of the pea aphid and the detailed relationship between trehalose level and feeding behaviour is still unclear. Therefore, a more comprehensive study of trehalose level and feeding behaviour is necessary because sugars are the main components of plant sap that aphids feed on, and would lay a solid foundation for further investigation of molecular biology and physiology of this pest.

In this study, we report (1) the effect of high sugar diets and knockdown of TPS and TRE expressions on the body trehalose and glucose contents of red and green A. pisum, (2) the stylet activity thus feeding behaviour of these treated aphids, and (3) the relationships between A. pisum fooding behaviour and the level of body trehalose and glucose. The results help to provide a theoretical basis for further development of biological agents targeting the feeding behaviours against $A$. pisum.

\section{Results}

Effect of RNAi and high sugar diets on ApTPS and ApTRE expression. Figure 1 shows the effects of the RNAi and high sugar (trehalose and sugar) diets on the expression level of ApTPS and ApTRE genes in both red and green of the $A$. pisum at $24 \mathrm{~h}$ and $48 \mathrm{~h}$ on fresh leaves after the RNAi treatments relative to the expression levels in the $A$. pisum treated with the normal diet. The ApTPS expression was significantly decreased for both red and green A. pisum at $24 \mathrm{~h}$ and $48 \mathrm{~h}$ after the ds TPS RNAi treatment (Fig. 1A), and was decreased at $24 \mathrm{~h}$ but increased significantly at $48 \mathrm{~h}$ after the ds TRE RNAi treatment by more than 2 folds in both biotypes compared with that in dsGPF-treated A. pisum (Fig. 1A). However, the ApTRE expression was downregulated by both dsTPS- and dsTRE-treatment relative to that in the dsGFP-treated A. pisum (Fig. 1B). 
The ApTPS expression was downregulated at $24 \mathrm{~h}$ and upregulated at $48 \mathrm{~h}$ on fresh leave after the high trehalose diet treatment for both biotypes (Fig. 1A). After the high glucose diet treatment, the ApTPS expression was upregulated only at $48 \mathrm{~h}$ on fresh leave for the green biotype (Fig. 1A). The APTRE expression was downregulated by the high sugar diets in most cases (Fig. 1B), apart from in the high trehalose-treated red biotype $A$. pisum where the ApTRE expression was upregulated at $24 \mathrm{~h}$ on fresh leave after the treatment. Notably, unlike the red A. pisum, the survival rate of the green A. pisum was significantly decreased by the RNAi treatments $(P<0.001$, Fig. S1). The reproduction (the total number of the offspring) was significantly decreased by the dsTPS and dsTRE treatments relative to that of the ds GFP-treated parents and by the high sugar diets relative to that of the untreated A. pisum (CK) $(P<$ 0.001 , Fig. S2). The expression of ApTPS and ApTRE, survival and reproduction had a similar trend between red and green $A$. pisum.

Trehalose and glucose contents. The effect of the RNAi-treatment and the high sugar diets on the trehalose and glucose contents were determined in the red and green A. pisum. There was no difference in both the trehalose and glucose contents between untreated control $A$. pisum (CK) and the dsGFPtreated $A$. pisum (dsGFP) (Fig. 2). The trehalose contents were decreased in the dsTPS-treated $A$. pisum but increased in the dsTRE-treated $A$. pisum in all cases compared with those in CK and dsGFP-treated $A$. pisum (Fig. 2A). The glucose contents were decreased in the dsTPS- and dsTRE-treated A. pisum at both time points ( $24 \mathrm{~h}$ and $48 \mathrm{~h}$ ) for both red and green A. pisum (Fig. 2B). The trehalose contents were not influenced by the high trehalose diet but decreased by the high glucose diet (Fig. 2A). However, both high sugar diets increased the glucose contents (Fig. 2B). In addition, the content of trehalose and glucose had a similar trend between red and green $A$. pisum.

Feeding behavior. Figure 3 shows the feeding activities recorded as EPG waveforms when the A. pisum probe into plants and presented as the percentage of each EPG waveform, and an overview of the representative EPG waveforms of treatment and control A. pisum on both time points is shown in Fig. S36 . At $24 \mathrm{~h}$ on fresh leaves after the treatments, no change in any EPG waveform was found in the dsTPStreated and dsTRE-treated red $A$. pisum compared with the dsGFP-treated $A$. pisum, which was not different from those of the untreated A. pisum (CK) (Fig. 3A; Tab. S2A). In the green A. pisum, the percentage of E2 waveform was increased by the dsTPS-treatment and decreased by the dsTREtreatment (Fig. 3C; Tab. S2C). The high sugar diets decreased and increased the percentage of E2 waveform of the red and green $A$. pisum, respectively. The high sugar diets also increased the percentage of $\mathrm{G}$ waveform of the red A. pisum (Fig. 3A; Tab. S2E) and the percentage of $\mathrm{F}$ waveform of the green $A$. pisum (Fig. 3C; Tab. S2G).

After $48 \mathrm{~h}$ treatment, the dsTPS-treatment increased the percentage of E2 waveform and decreased the percentage of $F$ and $G$ waveforms compared with the CK (Fig. 3B and 3D; Tab. S2B and S2D), but the dsTRE-treatment did not affect the percentage of any waveform compared with the CK group (Fig. 3B and 3D; Tab. S2B and S2D). The high sugar diets had little effect on the EPG waveforms (Fig. 3B and 3D; Tab. $\mathrm{S} 2 \mathrm{~F}$ and $\mathrm{S} 2 \mathrm{H}$ ) of both the red and green A. pisum. In addition, the feeding behaviours had a similar trend between red and green $A$. pisum. 
Relationships between feeding behaviour and physiological sugar level. To illustrate the relationshops of physiological sugar levels (trehalose and glucose levels) on the feeding behaviour, the relationships between the physiological sugar levels and the percentages of EPG waveforms were assayed using multiple nonlinear regression at $48 \mathrm{~h}$. As Figure 3B and 3D shown that the EPG waveforms were huge difference between the each group at $48 \mathrm{~h}$. These data shown that the percentage of $\mathrm{np}$ waveform was increased at the low both trehalose and glucose levels and the high both trehalose and glucose levels, while was decrased at the low trehalose level and high glucose level and the high trehalose levels and low glucose level, and the regression equation was $\left(R^{2}=0.371\right.$, Fig. $\left.4 \mathrm{~A}\right)$. Where $z, x$, and $y$ are the percentage of the waveform, trehalose content and glucose content, respectively. The percentage of $C$ waveform was increased with trehalose content elevalted, which was firstly increased and then decreased with glucose content rised, and the regression equation was $\left(R^{2}=0.5302\right.$, Fig. $\left.4 \mathrm{~B}\right)$. The percentage of $\mathrm{E} 1$ waveform showed the same trend as did the percentage of np waveform, and the regression equation was and $\left(R^{2}=0.347\right.$, Fig. $\left.4 \mathrm{C}\right)$. Interestingly, the percentage of E2 waveform was increased at the level of low trehalose and low glucose, low trehalose and high glucose, high trehalose and low glucose, and high trehalose and high glucose, and the regression equation was $\left(R^{2}=0.6975\right.$, Fig. $\left.4 \mathrm{D}\right)$ and the minimum at the point $(0.44,0.090)$. The percentage of $G$ waveform was firstly increased and then decreased with trehalose content rised, which was increased with glucose content rised, and the egression equation was $\left(R^{2}=0.6670\right.$, Fig. $\left.4 \mathrm{E}\right)$. The percentage of $\mathrm{F}$ waveform was firstly increased and then decreased with trehalose content or/and glucose content rised, and the egression equation was $\left(R^{2}=0.6218\right.$, Fig. $\left.4 \mathrm{~F}\right)$.

Trehalose and glucose are the two main sugars in the insects hemolymph and they plays a important role in food-choice behaviour ${ }^{30}$. We assayed whether the balance of trehalose and glucose induce a foodchoice feeding behaviour in A. pisum. As was observed that the top or bottom contour each subgraph of Fig. 4. There was a small difference in the percentage of np, E1, and E2 waveforms occurred between high trehalose:glucose (T:G) ratio and low $\mathrm{T}: \mathrm{G}$ ratio, but decreased the percentage of np, E1, and E2 (Fig. $4 A, 4 C$, and 4D). Interestingly, the percentage of $C, F$ and, $G$ waveforms were increased at low, low, and high $\mathrm{T}$ :G ratio (Fig. 4B, 4E, and 4F), respectively.

\section{Discussion}

Sugar metabolism plays a critical role in aphids' adaptation under various environmental conditions. Our results showed that high trehalose-diet feeding did not increase $A$. pisum hemolymph trehalose content, but increased glucose content (Fig. 2). The trehalose contents were decreased by the high glucose diet (Fig. 2A) and the glucose contents were increased by both high sugar diets (Fig. 2B), suggesting that glucose may be crucial to the $A$. pisum growth and development. The $A$. pisum may utilize lower glucose level in the hemolymph to regulate survival, reproduction and feeding behavior, and so are sensitive to the change in the hemolymph glucose level, while trehalose is stored as an energy resource. The insects' body fluid balance is also influenced by intrinsic and extrinsic factors such as food, gluconeogenesis, and glycogenolysis ${ }^{6,14,21}$. It is possible that, when the trehalose content in A. pisum's hemolymph is very high, it would be hydrolyzed to produce glucose. Gluconeogenesis contributed greatly to hemolymph sugar in 
insects maintained on a low carbohydrate diet, but on a high carbohydrate diet, the hemolymph sugar was derived mainly from dietary carbohydrate, whereas the generation of amino acids was regulated post-ingestively 21,31 .

It was also observed that the high sugar diets did not only affect the survival rate but also reduced the reproduction of red and green $A$. pisum (Fig. S1 and S2). It was reported that high trehalose negatively affected food intake of $L$. decemlineata and Spodoptera exigua ${ }^{27,32}$. High sucrose diet decreased the consumption rate of $A$. pisum, and a low sucrose diet increased food ingestion of Ceratitis capitata female by $35 \%$ compared with the control ${ }^{22,33}$. Moreover, the trehalose rich reduced reproduction and growth in insects $27,32,34$, it was found that over-accumulation of trehalose reduced $L$. decemlineata survival $^{27}$ and Drosophila melanogaster adaptation ${ }^{34}$.

The RNAi of ApTPS decreased trehalose content of the A. pisum as in B. minax and L. decemlineata ${ }^{27,28}$. However, the trehalose contents were increased in the dsTRE-treated $A$. pisum in all cases compared with those in CK and dsGFP-treated A. pisum (Fig. 2A). This is contradictory to the report in S. exigua larvae ${ }^{32}$. Thus, the effects of RNAi of TRE on the glucose content may be different in different insect species. The glucose contents were decreased in the dsTPS- and dsTRE-treated $A$. pisum at both time points $(24 \mathrm{~h}$ and $48 \mathrm{~h}$ ) for both red and green $A$. pisum (Fig. 2B), further confirming the sensitive regulation of the glucose level in the $A$. pisum.

The EPG technique is a useful tool to detect the feeding behaviour of piercing-sucking insects ${ }^{35}$. The high sugar diets and the RNAi of APTRE did not significantly change the percentage of each EPG waveform. The difference in the percentage of EPG waveforms between treatment and control groups was observed only at $48 \mathrm{~h}$ (Fig. 3; Tab. S2). RNAi of ApTPS increased the percentage of E2 waveform and decreased the percentage of $\mathrm{F}$ and $\mathrm{G}$ waveforms. Overall, the A. pisum spent more time on $\mathrm{E} 2$ waveform (phloemfeeding) (Fig. 3). This is consistent with the phloem-feeding activity of the A. pisum for nutrients, such as sucrose and amino acid contained in their host plant ${ }^{36}$.

The measurements of trehalose and glucose contents and the feeding behaviours of A. pisum treated with RNAi and high sugar diets provided an unique opptunity to analyse the relationships of the physiological sugar levels with the feeding behaviours of $A$. pisum. The analysis showed that the low both trehalose and glucose levels increased the activity of non-probing phase (np) and phloem phase (E1 and E2) but the high both trehalose and glucose levels also increased non-probing phase and phloem phase (Fig. 4A, 4C, and 4D). Low physiological sugar level decreased np increased A. pisum phloemfeeding to obtain more carbohydrates in agreement with the analysis by the EPG recording (Fig. 3B and 3D). The increase of phloem-feeding time under the low hemolymph sugar levels is a sign that the $A$. pisum needed more carbohydrates to maintain its homeostasis. However, the high hemolymph sugar levels increased phoem-feeding may be due to the range of the model. Interestingly, the low T:G ratio increased the activity of probing phase (C) and stylet work phase (F; Fig. 4B and 4E), indicating that $A$. pisum spent more time feeding in the cell walls, intercellular spaces of vascular tissue, and the mesophyll 
as when $A$. pisum feed on resistant plants ${ }^{4,37-39}$. On the other hands, the increased $\mathrm{F}$ waveform of aphids was also reported to restore the stylets bundle ${ }^{4}$, or probably due to the differences in salivary components $^{38}$. The high $\mathrm{T}: \mathrm{G}$ ratio increased the activity of xylem ingestion phase $(F)$ to intaken more water to avoid dehydration (Fig. 4F).

Overall, the findings from this study suggested that the level and balance of trehalose and glucose $A$. pisum food-choice behavior. This is the first reported using the EPG technique to study the link of $A$. pisum physiological sugar level and feeding behaviour. It provides strong evidence that the feeding behaviour of the A. pisum is influenced by the level and balance of trehalose and glucose in the body.

In conclusion, this study shows that RNAi of ApTPS and high sugar diets can affect the trehalose and/or glucose content in the body of $A$. pisum (Fig.1). This allows to analyse the relationships between sugar contents and feeding behaviours under $A$. pisum physiological conditions. Both low trehalose and glucose levels increased the time of non-probing phase and phloem feeding phase, the low $T: G$ ratio increased the feeding time of probing phase and stylet work, and decreased xylem feeding time. Future research is now required to validate the mechanism of physiological sugar level regulated feeding behaviour.

\section{Materials And Methods}

Insect and culture conditions. Clones of red and green morphs of $A$. pisum were established from single virginiparous females. Samples were collected in 2017 from same Alfalfa plant Medicago sativa in field, Lanzhou, China, and reared on the fava bean Vicia faba in the laboratory. All plants and A. pisum cultures were reared in an artificial climate incubator at $20 \pm 1{ }^{\circ} \mathrm{C}, 70 \pm 10 \%$ relative humidity, with a photoperiod of $16 \mathrm{~h} \mathrm{~L}: 8 \mathrm{~h}$ D. Mature A. pisum were put on a fava bean leaf for $12 \mathrm{~h}$ and the resulting neonate nymphs, 0-12 $\mathrm{h}$ old, were used for experiments throughout this study.

RNA isolation and first-strand cDNA synthesis. Total RNA was isolated using TRizol reagent (BBI Life Sciences, Shanghai, China) following the manufacturer's instructions. The total quantity of extracted RNA was assessed using a micro-volume UV spectrophotometer (Quawell Q5000, Quawell, USA). The RNA integrity was confirmed further by $1 \%$ formaldehyde agarose gel electrophoresis. Total RNA was dissolved in $50 \mathrm{~mL}$ DEPC-water and stored at $-80^{\circ} \mathrm{C}$. The first-strand $\mathrm{cDNA}$ was synthesized using a FirstStrand cDNA Synthesis kit (BioTeke, Beijing, China) and stored at $-20^{\circ} \mathrm{C}$ for subsequent experiments.

Cloning of TPS and TRE cDNAs. The primer sets, TPS-F/R of ApTPS and TRE-F/R of ApTRE, were designed using the primer software Primer 5.0 (Premier Biosoft, Palo Alto, CA, USA) based on the TPS gene sequence (GENBANK accession: XM_001943581.5) and the TRE gene sequence (GENBANK accession: XM_003245847.4) of A. pisum. The primers of the green fluorescent protein gene (GFP, pET28a-EGFP, Miaolingbio, Wuhan, China) were referenced from Yang et al. ${ }^{16}$. These primers are listed in Table S1. The components of the PCR reaction mixture included $1.0 \mathrm{~mL}$ of the template $(1 \mathrm{ng} / \mathrm{mL}), 12.5$ $\mathrm{mL} 2 \times$ Power Tap PCR MasterMix (BioTeke, Beijing, China), $1.0 \mathrm{~mL}$ of each primer $(10 \mathrm{mmol} / \mathrm{mL})$, and 9.5 
$\mathrm{mL}$ Rnase-free $\mathrm{H}_{2} \mathrm{O}$ concentration for a final volume of $20 \mathrm{~mL}$. The PCR reaction conditions were predenatured at $95^{\circ} \mathrm{C}$ for $5 \mathrm{~min}$, followed by 35 cycles of $95^{\circ} \mathrm{C} / 45 \mathrm{~s}$ for denature $55^{\circ} \mathrm{C} / 45 \mathrm{~s}$ for annealing and $72^{\circ} \mathrm{C} / 1 \mathrm{~min}$ for extension, and then $10 \mathrm{~min}$ at $72^{\circ} \mathrm{C}$ for a final extension. PCR products were subjected to $1.0 \%$ agarose gel electrophoresis and purified by DNA gel extraction kit (BioTeke, Beijing, China). The purified DNA was ligated into the pMD18-T vector (TaKaRa, Dalian, China) and sequenced by Tsing Ke Biological Technology (Tsing Ke Biological Technology, Beijing, China) using the dideoxynucleotide method. The lengths of the resulting ApTPS, ApTRE, and GFP genes were $421 \mathrm{bp}, 416$ bp, and $688 \mathrm{bp}$, respectively.

dsRNA synthesis. Three pairs of primers (dsTPS-F/R, dsTRE-F/R and dsGFP-F/R), with the T7 RNA promoter sequence flanking the 5 '-end of each gene, were designed and synthesized (Tab. S1), and used to make the templates for in vitro dsRNA transcription via PCR. The dsRNAs were synthesized using the TranscriptAid T7 High Yield Transcription Kit (Thermo Scientific, Wilmington, DE, USA) according to the manufacturer's protocol ${ }^{40}$. The size of the dsRNA products was confirmed by electrophoresis on a $1.5 \%$ agarose gel and the concentration was assessed using a micro-volume UV spectrophotometer.

dsRNA and high sugars diet treatments. The artificial diet bioassay was performed according to the following procedure ${ }^{41}$. A liquid artificial diet was prepared as described previously ${ }^{42,43}$, filtered through a $2 \mathrm{~mm}$ membrane, dispensed in $1.0 \mathrm{~mL}$ aliquots, and stored at $-20^{\circ} \mathrm{C}$ before assays. The testing diets were prepared by adding either each of dsRNA (dsTPS, dsTRE and dsGFP) or each of sugar (trehalose and glucose) to the $1.0 \mathrm{~mL}$ artificial diet for a final concentration of $400 \mathrm{ng} / \mathrm{mL}$ (dsRNA) and $100 \mathrm{mg} / \mathrm{mL}$ (sugar). The diet containing nuclease-free water was used as control of the high sugar diet treatments and diet containing dsGFP was used as control of the RNAi treatments. There was a total of 6 treatments including two controls for either red or green $A$. pisum.

Glass vials ( $2.5 \mathrm{~cm}$ in diameter) were sterilized for the aphid artificial double-membrane feeding assay and one opening was completely sealed with parafilm. Seventy microliters of the testing diet were placed on the parafilm and covered with parafilm. So the testing diet was sandwiched between two layers of the parafilm membrane at one opening of the glass vials ${ }^{44}$. The control group was fed with only the artificial diet without dsRNA or sugars.

Fifteen 3-day-old A. pisum were introduced into one vial, and the vial was closed with a piece of sterilized gauze as one of bioassays. The artificial diet was replaced every other day to prevent dsRNA degradation. After 4 days, all surviving $A$. pisum were transferred to fresh bean leaf discs.

Quantification of gene expression levels after RNAi treatments. Seven A. pisum were collected from fresh bean leaf discs at $24 \mathrm{~h}$ and $48 \mathrm{~h}$ after the 4-day treatment with the testing diet containing each of dsRNAs. A. pisum were immediately frozen in liquid nitrogen and three replicates were carried for each treatment. Total RNA was isolated from the seven pooled whole $A$. pisum bodies. The first-strand cDNA was synthesized from total RNA using a First-Strand cDNA Synthesis kit (BioTeke, Beijing, China). The RTqPCR analysis was carried out in $96-$ well $0.1-\mathrm{mL}$ block plates using a QuantStudio ${ }^{\mathrm{TM}} 5$ system (Thermo 
Scientific, Wilmington, DE, USA). Each reaction contained $1.0 \mathrm{~mL}$ of the cDNA template, $10.0 \mathrm{~mL} 2 \times$ Plus SYBR real-time PCR mixture (BioTeke, Beijing, China), $0.5 \mathrm{~mL}$ of each primer $(10 \mathrm{mmol} / \mathrm{mL}), 8 \mathrm{~mL}$ EDPC$\mathrm{ddH}_{2} \mathrm{O}$, and $0.5 \mathrm{~mL} 50 \times$ ROX Reference Dye concentration for a final volume of $20 \mathrm{~mL}$. The RT-qPCR reaction conditions were pre-denatured at $94^{\circ} \mathrm{C}$ for $2 \mathrm{~min}$, by 40 cycles of $94^{\circ} \mathrm{C} / 15 \mathrm{~s}$, and $55-62^{\circ} \mathrm{C} / 30 \mathrm{~s}$ for annealing. After each reaction, a melting curve analysis (denatured at $95^{\circ} \mathrm{C}$ for $15 \mathrm{~s}$, annealed at $60^{\circ} \mathrm{C}$ for $1 \mathrm{~min}$, and denatured at $95^{\circ} \mathrm{C}$ for $15 \mathrm{~s}$ ) was conducted to ensure consistency and specificity of the amplified product. Three biological replicates and three technical replicates were set for each treatment in the RT-qPCR analysis. Quantification of the transcript level was conducted according to the method ${ }^{45}$, and the ribosomal protein $L 27$ gene ( $r p L 27)$ was used as a reference gene $e^{46}$.

Trehalose and glucose content assays after high sugar diet treatments. Ten A. pisum were collected from fresh bean leaf discs at $24 \mathrm{~h}$ and $48 \mathrm{~h}$ after the 4-day treatment with the testing diet containing each of sugars. A. pisum were immediately frozen in liquid nitrogen and three replicates were carried for each treatment. The trehalose content assay was conducted according to the method described by Yang et al. ${ }^{16}$. Briefly, ten whole $A$. pisum bodies were ground in phosphate-buffered saline (PBS: $130 \mathrm{mM} \mathrm{NaCl} 7$ $\mathrm{mM} \mathrm{Na} 2 \mathrm{HPO}_{4} \cdot 2 \mathrm{H}_{2} \mathrm{O} ; 3 \mathrm{mM} \mathrm{NaH} \mathrm{PO}_{4} \cdot 2 \mathrm{H}_{2} \mathrm{O} ; \mathrm{pH} 7.0$ ), and then a $25 \mathrm{~mL}$ of tissue was taken and uniformly mixed with $25 \mathrm{~mL}$ of $1 \%$ sulfuric acid. The mixture was incubated at $90^{\circ} \mathrm{C}$ for $10 \mathrm{~min}$ and placed in ice for $3 \mathrm{~min}$, and then $25 \mathrm{~mL}$ of $30 \%$ potassium hydroxide solution was added into the sample and mixed uniformly. The resultant mixture was incubated at $90^{\circ} \mathrm{C}$ for $10 \mathrm{~min}$ and then in ice for $3 \mathrm{~min}$. Finally, 500 $\mathrm{mL}$ of $0.2 \%$ anthrone reagent was added to the sample and incubated at $90^{\circ} \mathrm{C}$ for $10 \mathrm{~min}$ and then in ice for $3 \mathrm{~min}$. The trehalose content was assayed by measuring the absorbance of the final reaction mixture at $630 \mathrm{~nm}$. The glucose content was determined using the glucose assay kit (Solarbio Biochemical Assay Division, Beijing, China) according to the manufacturer's protocols.

Evaluation of $\boldsymbol{A}$. pisum feeding behavior. The probing behaviour was evaluated with the electrical penetration graph (EPG) using an 8-channel DC-EPG device (Wageningen University, the Netherlands). Eight plants were placed in a faraday cage, and wingless $A$. pisum were placed on the abaxial side of the second fully expanded leaf from the top. Before exposure A. pisum to the plant, a 6 to $8 \mathrm{~cm}$ long gold wire (diameter $18 \mathrm{~mm}$ ) was conductively glued (water-based silver glue) to A. pisum dorsum as the recording electrode. The other end of the gold wire was attached to a $3 \mathrm{~cm}$ long copper wire (diameter $0.2 \mathrm{~mm}$ ) which was connected to the first head stage on the DC-EPG amplifier with the setting of 1 Giga-Ohm input resistance and 50xgain. The reference electrode was inserted into the soil and connected to the plant voltage output of the DC-EPG device. A. pisum from each treatment were randomly distributed during recording. For each treatment, only the $A$. pisum that showed activities in an $8 \mathrm{~h}$ recording period were considered as valid replicates.

The EPG signal was recorded by the Stylet+d software and the EPG waveforms were recognized and labeled using Stylet+av01.30 software (EPG Systems, Wageningen, Netherlands). The EPG parameters were calculated for each A. pisum treatment using the Excel workbook for automatic parameter 
calculation of EPG data 4.4.3 47,48 and then the means and standard errors of the mean (SEM) were calculated for each treatment at $24 \mathrm{~h}$ and $48 \mathrm{~h}$ on fresh bean leaf discs after 4-day treatments.

Survival and reproduction assays. A. pisum were reared on fresh bean leaf discs after the treatments in an artificial climate incubator at $20 \pm 1{ }^{\circ} \mathrm{C}, 70 \pm 10 \%$ relative humidity, with a photoperiod of $16 \mathrm{~h} \mathrm{~L}: 8 \mathrm{~h} \mathrm{D}$. Survival and reproduction assays were conducted for the control and treated $A$. pisum. The daily numbers of adult $A$. pisum deaths and newborn nymphs per adult $A$. pisum were recorded until they no longer produced nymphs, once per day starting from the first day after the treatments.

Multiple nonlinear regression. The $48 \mathrm{~h}$ trehalose and glucose content data of the A. pisum obtained in 2.7 and the percentage of EPG waveforms data of the A. pisum obtained in 2.8. The relationshops between the percentage of EPG waveforms under each treatment (z) and the corresponding physiological trehalose content $(\mathrm{x})$ and glucose content $(\mathrm{y})$ were then analyzed as by nonlinear curve fitting with the softwave 1stOpt 15.0 (7D-D oft High Technology Inc, China), where $a_{1}, a_{2}, a_{3}, a_{4}$, and $a_{5}$ : coefficient; $b$ : constant.

Statistical analysis. All statistical analyses were performed using 1stOpt 15.0, SPSS 19.0, and Origin 8.5 were used to construct the histograms. The RT-qPCR and sugar data were analyzed by Student's $t$-test. The EPG data (Tab. S2) and the total reproduction data (Fig. S2) were analyzed using one-way analysis of variance (ANOVA) followed by the Tukey's post hoc test. The survival data were subjected to a KaplanMeier survival log-rank analysis (Fig. S1) ${ }^{49}$. A $p$-value $<0.05$ was considered statistically significant.

\section{Declarations}

\section{Acknowledgements}

This work has supported by the National Natural Science Foundation of China $(31960351,31660522)$ and the Discipline Construction Fund Project of Gansu Agricultural University (GAU-XKJS-2018-149).

\section{Author's contributions}

G.W., Y.-P.G. and C.-Z.L. designed the research. G.W., Y.L., Y.-P.G. and P.Q. conducted the experiments. G.-W., C.-Z.L. and J.-J.Z. analysed the data. G.W. wrote the first draft of the manuscript and J.-J.Z. made critical revisions of the manuscript. All authors read and approved the manuscript.

\section{Acknowledgements}

We thank Fu-Qiang Luo, Yan Li, Xiao-Wei Li, Yin-Fang Zhang and Yong-Zhang Bai reared fava bean and $A$. pisum during the fieldwork. We also like to thank Jeffrey A. Coulter of Department of Agronomy and Plant Genetics, University of Minnesota for a critical review and helpful suggestions for this article.

\section{Additional Information}


Conflicts of interest: The authors declare no conflict of interest.

Publisher's note: Springer Nature remains neutral with regard to jurisdictional claims in published maps and institutional affiliations.

\section{References}

1. Azzouz, H., Giordanengo, P., WCkers, F. L. \& Kaiser, L. Effects of feeding frequency and sugar concentration on behavior and longevity of the adult aphid parasitoid: Aphidius ervi (Haliday) (Hymenoptera: Braconidae). Bioll. Control.31, 445-452 (2004).

2. Abisgold, J. D., Simpson, S. J. \& Douglas, A. E. Nutrient regulation in the pea aphid Acyrthosiphon pisum: application of a novel geometric framework to sugar and amino acid consumption. Physiol. Entomol.19, 95-102 (1994).

3. Jacobson, A. L. \& Kennedy, G. G. Electrical penetration graph studies to investigate the effects of cyantraniliprole on feeding behavior of Myzus persicae (Hemiptera: Aphididae) on Capsicum annuum. Pest Manag. Sci. 70, 836-840 (2014).

4. Machado-Assefh, C. R. \& Alvarez, A. E. Probing behavior of aposymbiotic green peach aphid (Myzus persicae) on susceptible Solanum tuberosum and resistant Solanum stoloniferum plants. Insect Sci. 25, 127-136 (2018).

5. Chen, C., Ye, S., Hu, H., Xue, C. \& Yu, X. Use of electrical penetration graphs (EPG) and quantitative PCR to evaluate the relationship between feeding behaviour and Pandora neoaphidis infection levels in green peach aphid, Myzus persicae. J. Insect Physiol. 104, 9-14 (2017).

6. Cao, H. H., Zhang, Z. F., Wang, X. F. \& Liu, T. X. Nutrition versus defense: Why Myzus persicae (green peach aphid) prefers and performs better on young leaves of cabbage. PLoS One.13, e0196219 (2018).

7. Elisa, G. et al. Electrical penetration graph technique as a tool to monitor the early stages of aphid resistance to insecticides. Pest Manag. Sci.72, 707-718 (2016).

8. Tjallingii, F. et al. Fine structure of aphid stylet routes in plant tissues in correlation with EPG signals. Physiol. Entomol.18, 317-328 (1993).

9. Spiller, N. J., Koenders, L. \& Tjallingii, W. F. Xylem ingestion by aphids-a strategy for maintaining water balance. Entomol. Exp. Appl.55, 101-104 (2011).

10. Sauvion, N., Charles, H., Febvay, G. \& Rahbé, Y. Effects of jackbean lectin (ConA) on the feeding behaviour and kinetics of intoxication of the pea aphid, Acyrthosiphon pisum. Entomol. Exp. Appl.110, 31-44 (2010).

11. Elbein, A. D. New insights on trehalose: a multifunctional molecule. Glycobiology.13, 17R-27R (2003).

12. Arakane, Y. \& Muthukrishnan, S. Insect chitinase and chitinase-like proteins. Cell Mol. Life Sci. 67, 201-216 (2010). 
13. Ekta, S., Thorat, L. J., Nath, B. B. \& Gaikwad, S. M. Insect trehalase: Physiological significance and potential applications. Glycobiology. 25, 357-367 (2015).

14. Thompson, S., Borchardt, D. \& Wang, L. Dietary nutrient levels regulate protein and carbohydrate intake, gluconeogenic/glycolytic flux and blood trehalose level in the insect Manduca sexta L. J. Comp. Physiol. B.173, 149-163 (2003).

15. Shen, Q. D. et al. Excess trehalose and glucose affects chitin metabolism in brown planthopper (Nilaparvata lugens). J. Asia. Pac. Entomol.20, 449-455 (2017).

16. Yang, M. M. et al. Knockdown of two trehalose-6-phosphate synthases severely affects chitin metabolism gene expression in the brown planthopper Nilaparvata lugens. Pest Manag. Sci.73, 206216 (2017).

17. Becker, A., Schlöder, P., Steele, J. E. \& Wegener, G. The regulation of trehalose metabolism in insects. Experientia.52, 433-439 (1996).

18. Matsuda, H., Yamada, T., Yoshida, M. \& Nishimura, T. Flies without Trehalose. J. Biol. Chem.290, 1244-1255 (2015).

19. Vanin, S., Bubacco, L. \& Beltramini, M. Seasonal variation of trehalose and glycerol concentrations in winter snow-active insects. Cryo Letters.29, 485-491 (2008).

20. Simpson, S. J. \& Raubenheimer, D. The central role of the haemolymph in the regulation of nutrient intake in insects. Physiol. Entomol.18, 395-403(1993).

21. Thompson, S. N. Pyruvate cycling and implications for regulation of gluconeogenesis in the insect, Manduca sexta L. Biochem. Biophys. Res. Commun.274, 787-793 (2000).

22. Simpson, S. J., Abisgold, J. D. \& Douglas, A. E. Response of the pea aphid (Acyrthosiphon pisum) to variation in dietary levels of sugar and amino acids: the significance of amino acid quality. J. Insect. Physiol.41, 71-75 (1995).

23. Asaf, S. et al. Osmoprotective functions conferred to soybean plants via inoculation with Sphingomonas sp. LK11 and exogenous trehalose. Microbiol. Res. 205, 135-145 (2017).

24. Yu, W., Zhao, R., Wang, L., Zhang, S. \& Shen, L. ABA signaling rather than ABA metabolism is involved in trehalose-induced drought tolerance in tomato plants. Planta.250, 643-655 (2019).

25. Kramer, K. J. \& Koga, D. Insect chitin $\bigotimes$ Physical state, synthesis, degradation and metabolic regulation. Insect Biochem.16, 851-877 (1986).

26. Chen, J. et al. Feeding-based RNA interference of atrehalose phosphate synthasegene in the brown planthopper, Nilaparvata lugens. Insect Mol. Biol. 19, 777-786 (2010).

27. Shi, J. F. et al. Physiological roles of trehalose in Leptinotarsa larvae revealed by RNA interference of trehalose-6-phosphate synthase and trehalase genes. Insect Biochem. Mol. Biol.77, 52-68 (2016).

28. Xiong, K. C. et al. RNA interference of a trehalose-6-phosphate synthase gene reveals its roles during larval-pupal metamorphosis in Bactrocera minax (Diptera: Tephritidae). J. Insect Physiol. 91-92, 8492 (2016). 
29. Wang, G., Gou, Y., Guo, S., Zhou, J. J. \& Liu, C. RNA interference of trehalose-6-phosphate synthase and trehalase genes regulates chitin metabolism in two color morphs of Acyrthosiphon pisum Harris. Sci. Rep.11, 948 (2021).

30. Dus, M., Min, S., Keene, A., Lee, G. \& Suh, G. Taste-independent detection of the caloric content of sugar in Drosophila. Proc. Natl. Acad. Sci. U. S. A.108, 11644-11649 (2011).

31. Thompson, S. N. Long-term regulation of glucogenesis by dietary carbohydrate and relevance to blood sugar level in an insect Manduca sexta L. Int. J. Biochem. Cell Biol.30, 987-999 (1998).

32. Chen, J. et al. Different functions of the insect soluble and membrane-bound trehalase genes in chitin biosynthesis revealed by RNA interference. Plos One.5, e10133 (2010).

33. Canato, C. M. \& Zucoloto, F. S. Feeding behavior of Ceratitis capitata (Diptera, Tephritidae): influence of carbohydrate ingestion. J. Insect Physiol.44, 149-155 (1998).

34. Yasugi, T., Yamada, T. \& Nishimura, T. Adaptation to dietary conditions by trehalose metabolism in Drosophila. Sci. Rep.7, 1619 (2017).

35. Civolani, S. et al. Stylet penetration of Cacopsylla pyri; an electrical penetration graph (EPG) study. J. Insect Physiol.57, 1407-1419 (2011).

36. Douglas, A. E. The nutritional physiology of aphids. Adv. In Insect Phys.31, 73-140 (2003).

37. Montllor, C. B. \& Tjallingii, W. F. Stylet penetration by two aphid species on susceptible and resistant lettuce. Entomol. Exp. Appl.52, 103-111 (2011).

38. Machado-Assefh, C. R., Lopez-Isasmendi, G., Tjallingii, W. F., Jander, G. \& Alvarez, A. E. Disrupting Buchnera aphidicola, the endosymbiotic bacteria of Myzus persicae, delays host plant acceptance. Arthropod-Plant Inte.9, doi: 10.1007/s11829-015-9394-8 (2015).

39. Sun, M., Voorrips, R. E. \& Vosman, B. Aphid populations showing differential levels of virulence on Capsicum accessions. Insect Sci.27, 336-348 (2020).

40. Yang, W. J. et al. Functional characterization of chitin deacetylase 1 gene disrupting larval-pupal transition in the drugstore beetle using RNA interference. Comp. Biochem. Physiol. B. Biochem. Mol. Biol.219-220B, 10-16 (2018).

41. Zhang, M. et al. Identifying potential RNAi targets in grain aphid (Sitobion avenae F.) based on transcriptome profiling of its alimentary canal after feeding on wheat plants. Bmc Genomics14, 560 (2013).

42. Febvay et al. Influence of the amino acid balance on the improvement of an artificial diet for a biotype of Acyrthosiphon pisum (Homoptera: Aphididae). Can. J. Zool.66, 2449-2453 (1988).

43. Sapountzis, P. et al. New insight into the RNA interference response against cathepsin-L gene in the pea aphid, Acyrthosiphon pisum: Molting or gut phenotypes specifically induced by injection or feeding treatments. Insect Biochem. Mol. Biol.51, 20-32 (2014).

44. Xia, W. K. et al. Functional analysis of a chitinase gene during the larval-nymph transition in Panonychus citri by RNA interference. Exp. Appl. Acarol.70, 1-15 (2016). 
45. Livak, K. \& Schmittgen, T. Analysis of relative gene expression data using real-time quantitative PCR and the 2(-Delta Delta C(T)) Method. Methods.25, 402-408 (2001).

46. Mutti, N. S., Yoonseong, P., Reese, J. C. \& Reeck, G. R. RNAi knockdown of a salivary transcript leading to lethality in the pea aphid, Acyrthosiphon pisum. J. Insect Sci. 6, 1-7 (2006).

47. Ebert, T. A. et al. A new SAS program for behavioral analysis of electrical penetration graph data. Comput. Electron. Agric.116, 80-87 (2015).

48. Sarria, E., Cid, M., Garzo, E. \& Fereres, A. Excel Workbook for automatic parameter calculation of EPG data. Comput. Electron. Agric.67, 35-42 (2009).

49. Xiao, L., Ming-Jing, Q., Yi, Z., Jian-Wen, L. \& Tong-Xian, L. Expression of neuropeptide F gene and its regulation of feeding behavior in the pea aphid, Acyrthosiphon pisum. Front. Physiol.9, 87 (2018).

\section{Figures}
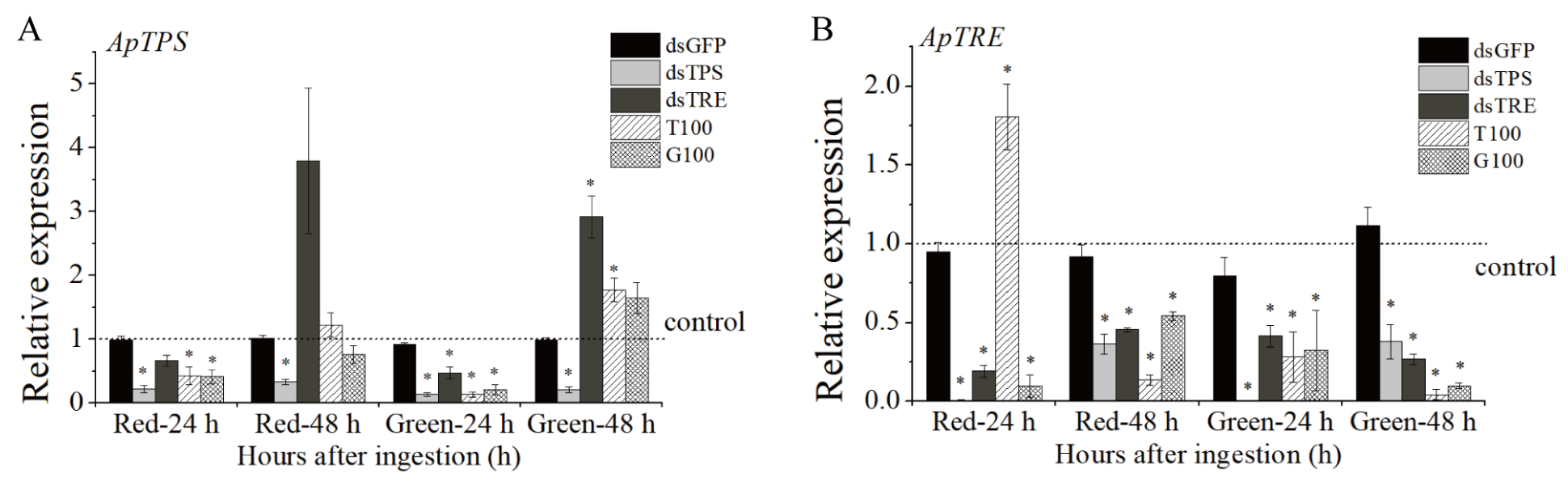

Figure 1

The expression levels of ApTPS (A) and ApTRE (B) genes. The expression level was represented relative to those of dsGFP-treated $A$. pisum as fold change and presented as Means \pm SEM of three replicates. All data were analyzed using Student's t-test. The asterisk indicates significant differences between treatment and control ( $\left.{ }^{*} P<0.05\right)$. A. pisum treated with RNAi of GFP gene (dsGFP), A. pisum treated with RNAi of TPS gene (dsTPS), A. pisum treated with RNAi of TRE gene (dsTRE), A. pisum treated with high trehalose diet (T100), A. pisum treated with high glucose diet (G100) and A. pisum fed with normal diet (control). 
A

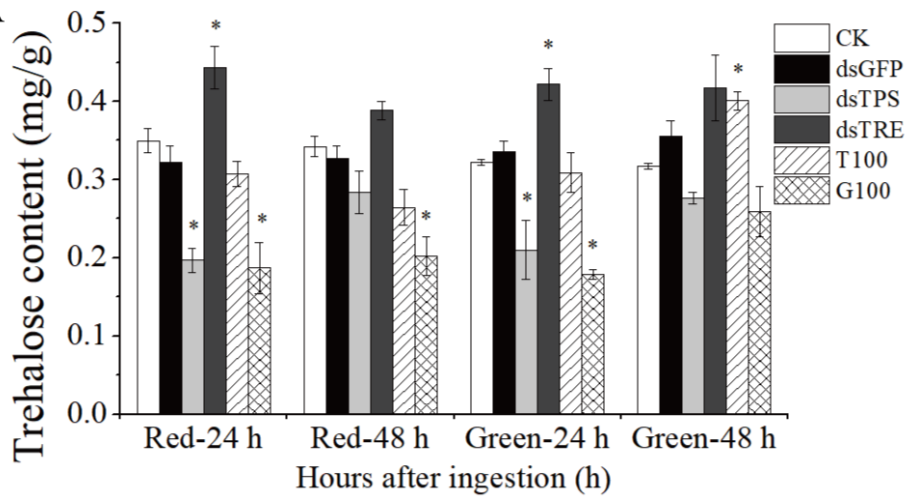

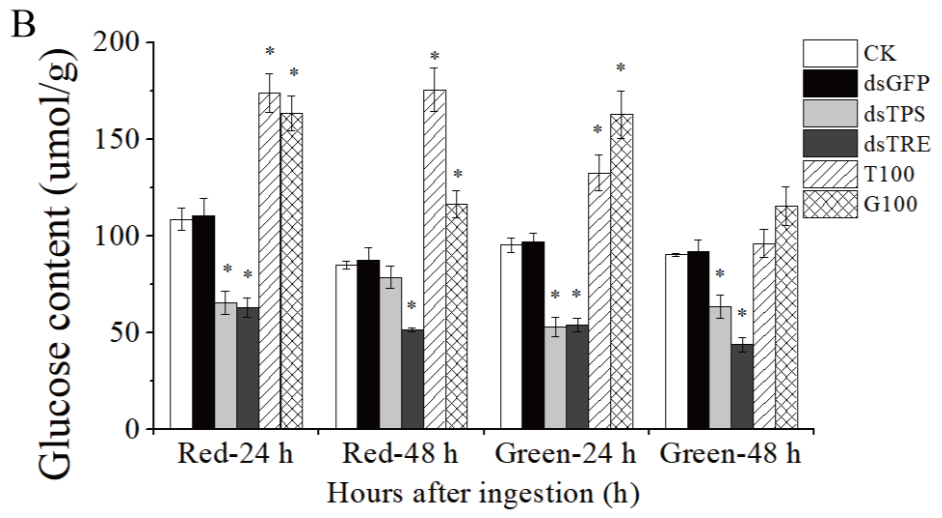

Figure 2

The physiological content of trehalose (A) and glucose (B) in red and green biotypes of the A. pisum. The content of trehalose and glucose were presented as Means \pm SEM of three replicates. A. pisum treated with RNAi of GFP gene (dsGFP), A. pisum treated with RNAi of TPS gene (dsTPS), A. pisum treated with RNAi of TRE gene (dsTRE), A. pisum treated with high trehalose diet (T100), A. pisum treated with high glucose diet (G100) and A. pisum fed with normal diet (CK). All data were analyzed using Student's t-test. The asterisk indicates significant differences between treatment and control $\left({ }^{*} \mathrm{P}<0.05\right)$.

A

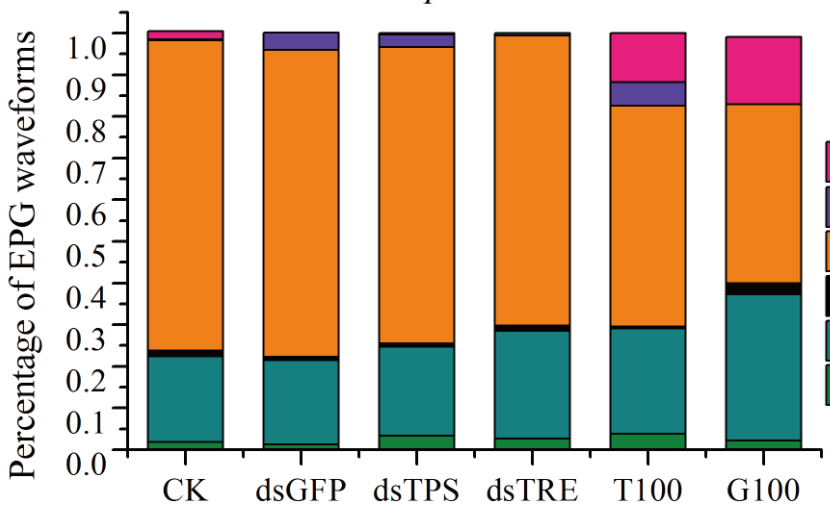

C

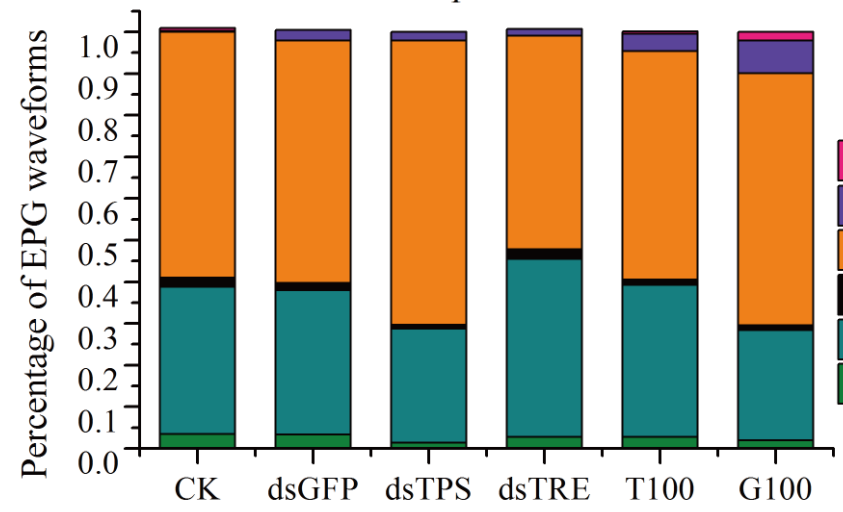

B

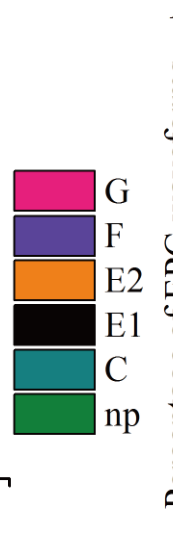

B

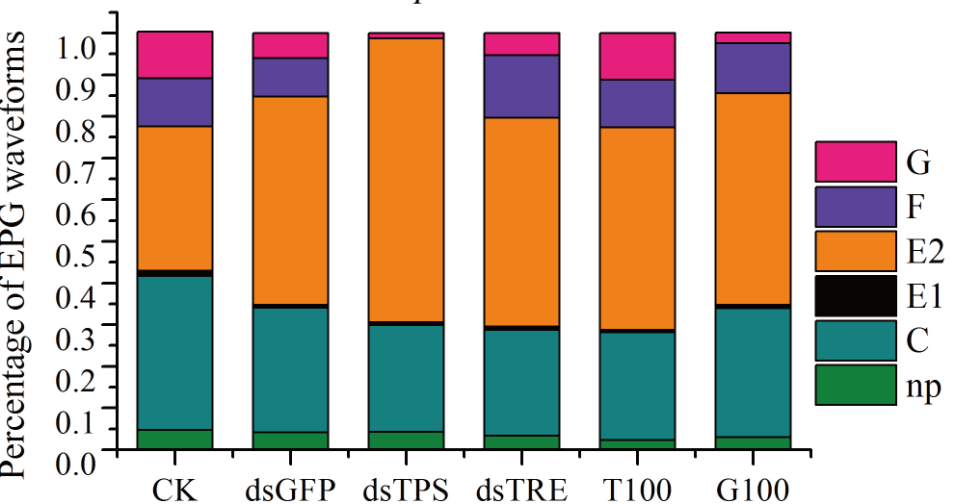

D

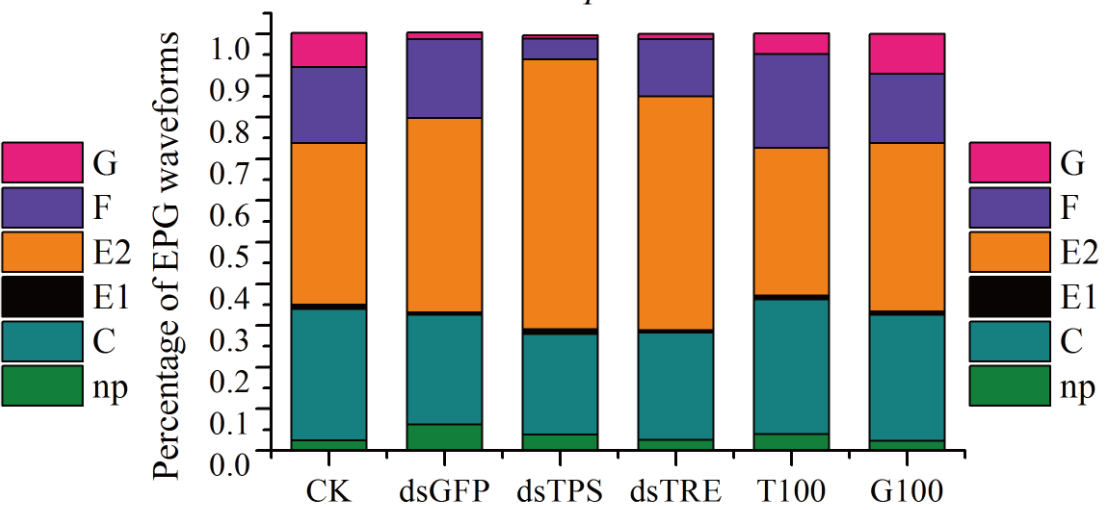

Figure 3 
Mean percentage of the EPG waveforms, during $8 \mathrm{~h}$ EPG recording. The percentage of EPG waveforms in red A. pisum at $24 \mathrm{~h}(\mathrm{~A})$ and $48 \mathrm{~h}(\mathrm{~B})$. The percentage of EPG waveforms in green A. pisum at $24 \mathrm{~h}(\mathrm{C})$ and $48 \mathrm{~h}(\mathrm{D})$. A. pisum treated with RNAi of GFP gene (dsGFP), A. pisum treated with RNAi of TPS gene (dsTPS), A. pisum treated with RNAi of TRE gene (dsTRE), A. pisum treated with high trehalose diet (T100), A. pisum treated with high glucose diet (G100) and A. pisum fed with normal diet (CK). The waveform for xylem ingestion $(G)$, waveform for derailed stylet mechanics $(F)$, waveform for phloem ingestion (E2), waveform for phloem salivation (E1), waveform for intercellular apoplastic stylet pathway (C) and non-probing (np). 
A $\quad \mathrm{z}=-0.0055 \mathrm{x}^{2}-0.63 \mathrm{y}^{2}+3.12 \mathrm{xy}-0.23 \mathrm{x}-0.84 \mathrm{y}+0.10$

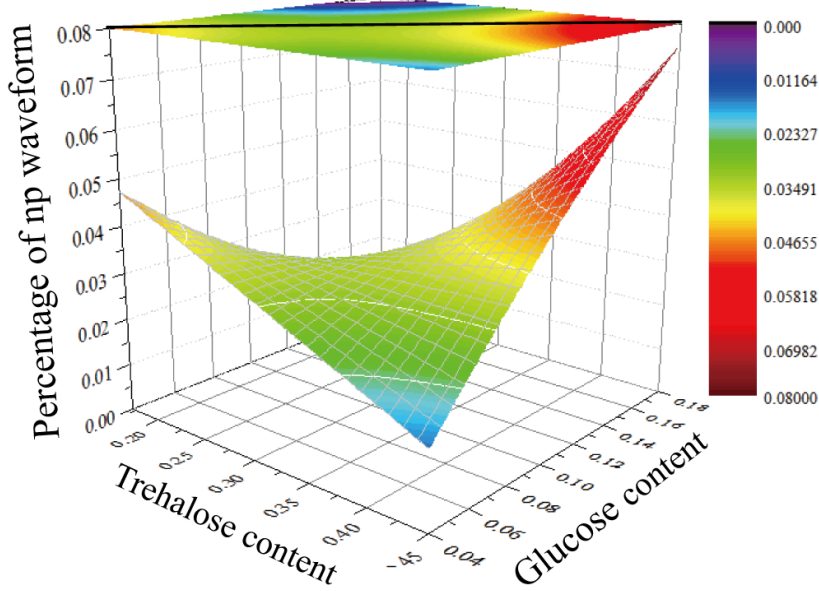

C

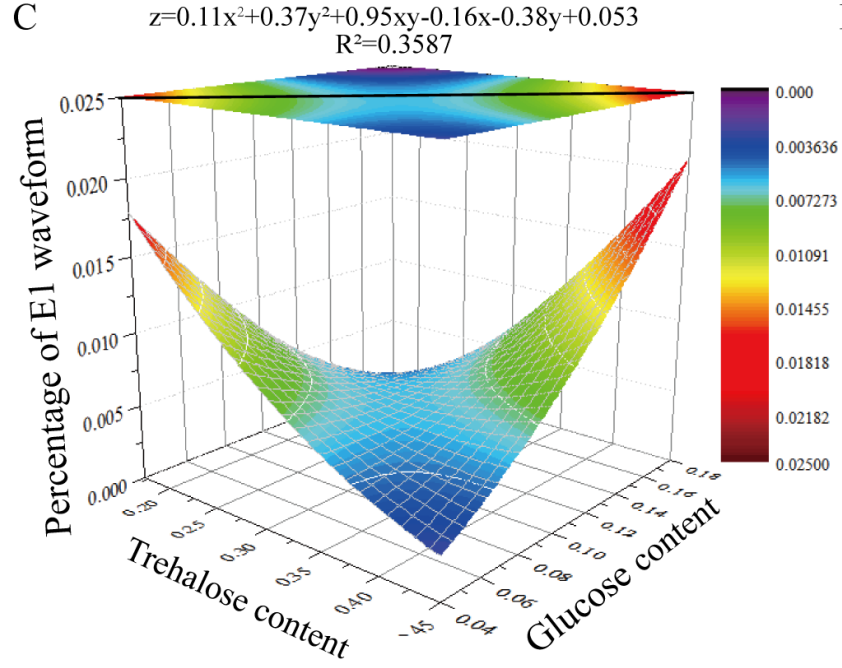

E

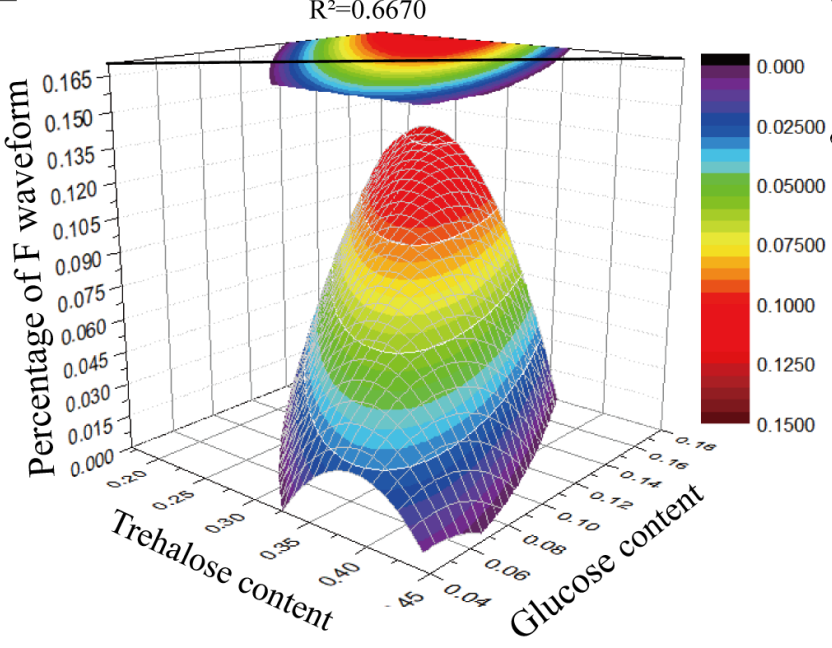

B $\quad \mathrm{z}=-1.10 \mathrm{x}^{2}-23.00 \mathrm{y}^{2}-8.91 \mathrm{xy}+1.73 \mathrm{x}-8.10 \mathrm{y}-0.42$

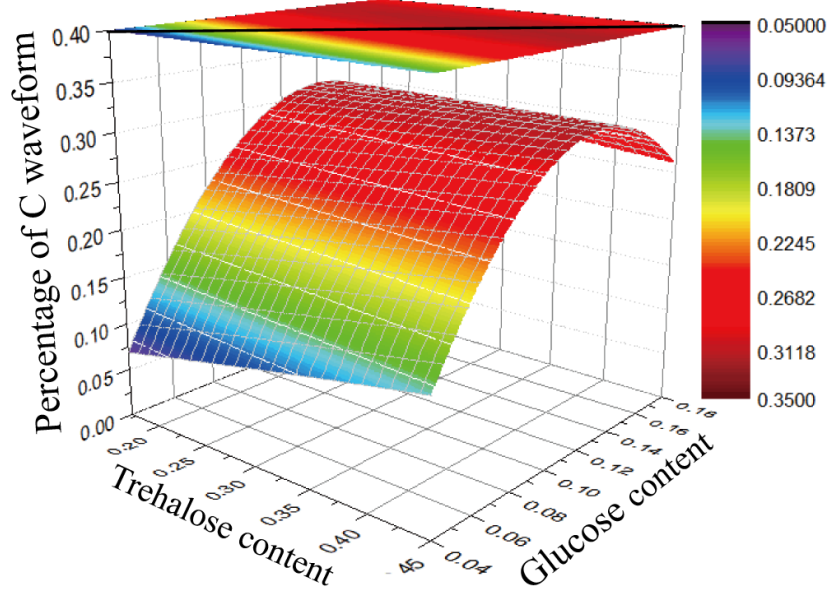

$\mathrm{D}$

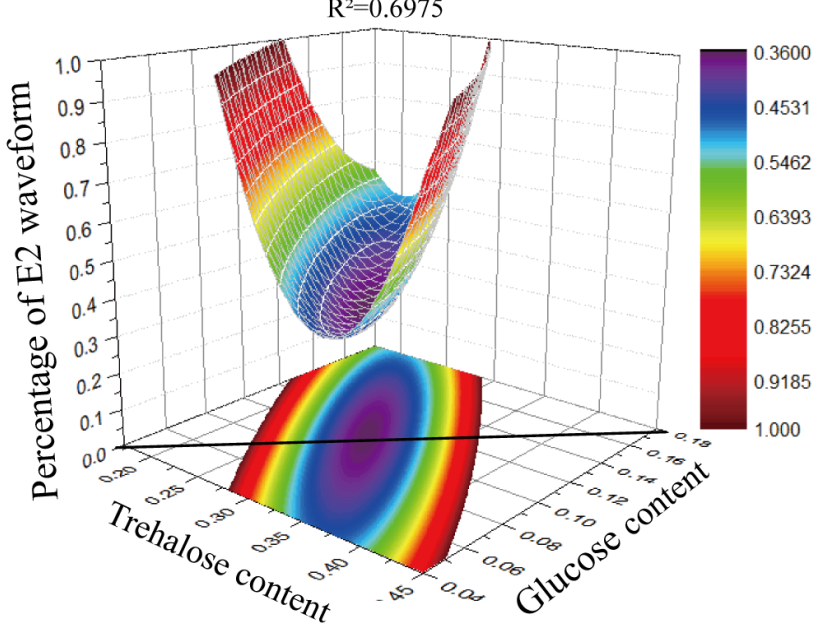

F $\quad \mathrm{z}=-6.90 \mathrm{x}^{2}-12.34 \mathrm{y}^{2}-16.52 \mathrm{xy}+6.04 \mathrm{x}+8.47 \mathrm{y}-1.34$

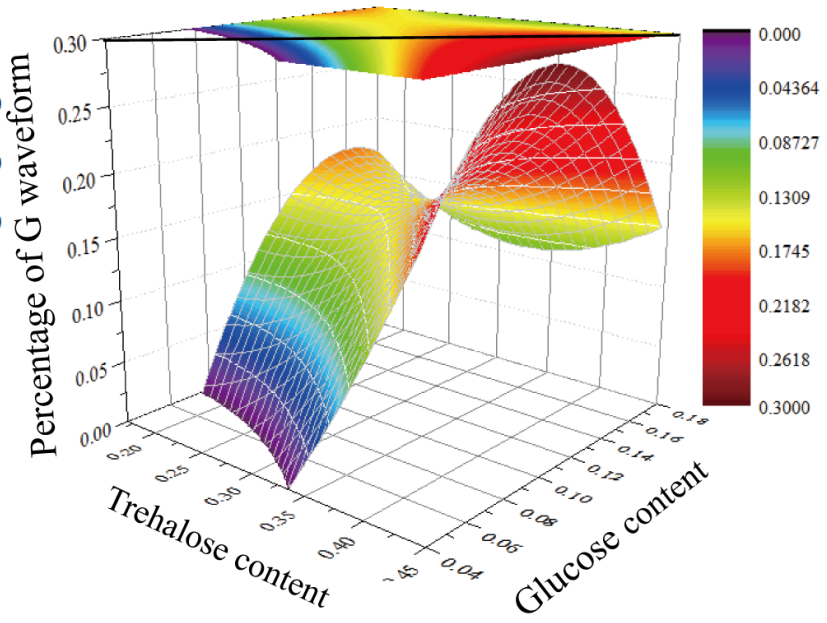

\section{Figure 4}

Relationship between feeding behaviour and the physiological sugar levels of A. pisum. The percentage of each waveform is the mean of three measurements at $48 \mathrm{~h}$ after each treatment (dsTPS, dsTRE, dsGFP, T100, G100 and normal diet). Z-axis: the percentage of EPG waveform by multiple nonlinear regression, where $\mathrm{x}$ is trehalose contents; $\mathrm{y}$ is glucose contents; $\mathrm{X}$-axis: trehalose content; $\mathrm{Y}$-axis: glucose content; The np waveform (A), C waveform (B), E1 waveform (C), E2 waveform (D),F wavefor (E), and G 
waveform (F). The coulor scale represents the percentage of EPG waveforms as in the Z-axis, top, and bottom contour.

\section{Supplementary Files}

This is a list of supplementary files associated with this preprint. Click to download.

- SupplementaryMaterials.pdf 